\title{
Pioneering studies on monogenic central precocious puberty
}

${ }^{1}$ Unidade de Endocrinologia do Desenvolvimento, Laboratório de Hormônios e Genética Molecular LIM/42 do Hospital das Clínicas, Disciplina de Endocrinologia da Faculdade de Medicina da Universidade de São Paulo, São Paulo, SP, Brasil

Correspondence to: Ana Pinheiro Machado Canton/ Ana Claudia Latronico, MD, PhD Hospital das Clínicas,

Faculdade de Medicina,

Universidade de São Paulo

Disciplina de Endocrinologia e

Metabologia

Av. Dr. Enéas de Carvalho Aguiar, 255,

$7^{\circ}$ andar, sala 7037

05403-900 - São Paulo, SP, Brasil

anapmc@usp.br

anacl@usp.br

Received on May/9/2019

Accepted on Jul/28/2019

DOI: 10.20945/2359-3997000000164
Ana Pinheiro Machado Canton'

https://orcid.org/0000-0001-8662-5412

Carlos Eduardo Seraphim ${ }^{1}$

https://orcid.org/0000-0002-4890-9470

Vinicius Nahime Brito ${ }^{1}$

https://orcid.org/0000-0003-4140-6296

Ana Claudia Latronico'

https://orcid.org/0000-0001-6782-693X

\begin{abstract}
Pubertal timing in humans is determined by complex interactions including hormonal, metabolic, environmental, ethnic, and genetic factors. Central precocious puberty (CPP) is defined as the premature reactivation of the hypothalamic-pituitary-gonadal axis, starting before the ages of 8 and 9 years in girls and boys, respectively; familial CPP is defined by the occurrence of CPP in two or more family members. Pioneering studies have evidenced the participation of genetic factors in pubertal timing, mainly identifying genetic causes of CPP in sporadic and familial cases. In this context, rare activating mutations were identified in genes of the kisspeptin excitatory pathway (KISS1R and KISS1 mutations). More recently, loss-of-function mutations in two imprinted genes (MKRN3 and DLK1) have been identified as important causes of familial CPP, describing novel players in the modulation of the hypothalamic-pituitary-gonadal axis in physiological and pathological conditions. MKRN3 mutations are the most common cause of familial CPP, and patients with MKRN3 mutations present clinical features indistinguishable from idiopathic CPP. Meanwhile, adult patients with DLK1 mutations present high frequency of metabolic alterations (overweight/obesity, early onset type 2 diabetes and hyperlipidemia), indicating that $D L K 1$ may be a novel link between reproduction and metabolism. Arch Endocrinol Metab. 2019;63(4):438-44
\end{abstract}

Keywords

Central precocious puberty; genetics; MKRN3; DLK1

\section{INTRODUCTION}

$\mathrm{P}$ ubertal development results from the re-emergence of pulsatile hypothalamic gonadotropin-releasing hormone $(\mathrm{GnRH})$ secretion, which is coordinated by a partially clarified mechanism involving inhibitory, stimulatory, and permissive factors acting upstream of GnRH neurons (1). Moreover, pubertal timing in humans is determined by complex interactions of different influences, such as metabolic, environmental, ethnic, and genetic factors (2). Genereally, it occurs between the ages of 8 and 13 in girls; and 9 and 14 in boys (2). Therefore, puberty is considered precocious if it starts prior to the ages of 8 and 9 in girls and boys, respectively (2).

The involvement of genetic factors in puberty control has been indicated by several evidences, such as similar age at menarche between mother and daughter, as well as among individuals of the same ethnic group; higher correlation in pubertal timing between monozygotic twins than between dizygotic twins or sibling pairs; and large-scale genome-wide association studies $(1,3)$. It is estimated that $60-80 \%$ of the variation in pubertal timing is due to genetic factors (1). Familial precocious puberty is typically defined by the occurrence of more than one affected family member (4).

Central precocious puberty (CPP) results from the premature activation of the hypothalamic-pituitarygonadal axis (HPG) and is considered "idiopathic" or of unknown causes, when congenital or acquired lesions in the central nervous system and monogenic defects are ruled out (2). Idiopathic CPP frequency varies according to population registries. In American girls, the incidence was estimated to be 1 in 5,000 to 10,000; however, in Danish girls, the prevalence was 1 in 500 (5). Regardless of the cohort, the prevalence of CPP is sexually dimorphic, being higher in girls than in boys (2). Up to now, Brazilian registries regarding population frequency of idiopathic CPP are unavailable. 
The occurrence of familial cases of CPP, not always detectable through anamnesis, fortifies the genetic basis of this disorder. De Vries and cols. (4) studied a large cohort of children with idiopathic CPP and identified a familial component in approximately $27 \%$ of them. Notably, inheritance was described in both maternal and paternal transmission in an autosomal dominant inheritance pattern with incomplete penetrance.

The diagnosis of CPP is based on physical exam findings indicating progressive puberty and on laboratory evaluation confirming central HPG axis activation, mainly through basal or GnRH-stimulated luteinizing hormone (LH) levels. After biochemical confirmation of CPP, a magnetic resonance imaging of the central nervous system must be performed in all patients to exclude anatomical abnormalities (2).

In recent years, genetic factors that regulate the HPG axis and modulate pubertal timing have been partially elucidated, primarily enabled by more sensitive and powerful molecular biology tools and epidemiological studies, such as large-scale genome-wide association studies, genomic microarray, whole exome sequencing, and, more recently, whole genome sequencing. This diagnostic advancement is revealing genetic causes of CPP involving novel players that modulates the HPG

Table 1. Genetic causes of central precocious puberty

\begin{tabular}{l}
\hline Monogenic causes \\
\hline 1. Gain-of-function (activating) mutations in KISS1R and KISS1 genes \\
(kisspeptin pathway genes) \\
2. Loss-of-function (inactivating) mutations in MKRN3 gene \\
3. Loss-of-function (inactivating) mutations in DLK1 gene \\
\hline Chromosomal abnormalities \\
\hline 1. Temple syndrome: maternal uniparental disomy, epimutation or paternal \\
deletion at chromosome 14q32.2 \\
2. Silver-Russell syndrome: maternal uniparental disomy of chromosome 7 \\
3. Williams-Beuren syndrome: $7 q 11.23$ deletion \\
4. Prader-Willi syndrome: maternal uniparental disomy or paternal deletion at \\
15q11-q13 \\
5. Rare cases of distinct copy number variants: 1p36 deletion, 9p distal \\
deletion, 9q34.3 duplication, Xp22.33 deletions, Xp11.23-p11.22 \\
duplication, and CDKL5 gene deletion
\end{tabular}

axis in physiological and pathological conditions (1) (Table 1). In this context, the Brazilian research group of pubertal disorders studies from the Developmental Endocrinology Unit at the São Paulo University Medical School has conducted pioneering studies with a prosperous collaboration from American researchers (Figure 1). Herein, we revise the current known genetic causes of CPP, emphasizing on monogenic causes.

\section{Monogenic causes of central precocious puberty (Table 2)}

\section{Activating mutations in genes KISS1 and KISS1R leading to sporadic CPP}

In 2008, the first monogenic defect in a patient with CPP was identified: a heterozygous activating mutation (p.Arg386Pro) of KISSIR gene, also called GPR54, which encodes the kisspeptin receptor (6). By biological plausibility, activating mutations of the kisspeptin pathway were expected to cause premature activation of the HPG axis, thus leading to CPP. This mutation was identified in an adopted girl presenting progressive thelarche since birth followed by accelerated growth, skeletal maturation, and development of progressive secondary sexual characters at age 7 . In vitro studies showed that the mutation led to prolonged activation of intracellular signaling pathways in response to kisspeptin $(2,6)$.

Parallel with the first kisspeptin pathway activating defect discovery, Silveira and cols. (7) identified an activating heterozygous mutation in the kisspeptin gene (KISSI) (p.Pro74Ser). This rare variant was identified in a boy who developed very early sporadic CPP at age 1 . His mother and maternal grandmother were carriers of the same mutation, despite normal pubertal development, thus suggesting an incomplete sexdependent penetrance of the phenotype. In vitro studies identified that the p.Pro74Ser mutation had a greater capacity to stimulate signal transduction than the wild type, leading to greater kisspeptin bioavailability $(2,7)$.

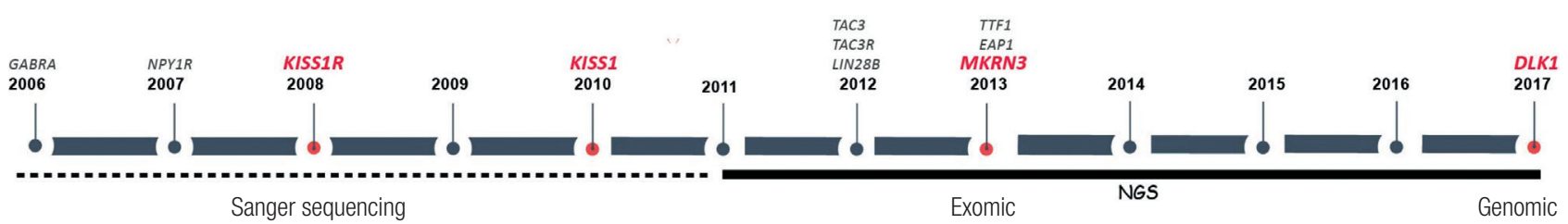

Figure 1. Timeline of the pioneering studies on monogenic central precocious puberty with the main participation of the Brazilian research group of pubertal disorders studies from the Developmental Endocrinology Unit at the São Paulo University Medical School. 
Table 2. Genetic and phenotypic characteristics of reported patients with monogenic causes of central precocious puberty

\begin{tabular}{|c|c|c|c|c|c|c|c|}
\hline \multirow[b]{2}{*}{ Gene } & \multirow{2}{*}{$\begin{array}{l}\text { Patients } \\
\text { reported }\end{array}$} & \multirow{2}{*}{$\begin{array}{l}\text { Genetic defects } \\
\text { (mutations) }\end{array}$} & \multirow[b]{2}{*}{ Inheritance } & \multicolumn{2}{|c|}{ Pubertal development, yr } & \multirow[b]{2}{*}{ Other clinical features } & \multirow[b]{2}{*}{ Ref. } \\
\hline & & & & Puberty onset & $\begin{array}{l}\text { Menarche } \\
\text { (untreated) }\end{array}$ & & \\
\hline KISS1R & $1(F)$ & Gain of-function missense & - & $\begin{array}{c}\text { Thelarche since birth } \\
\text { Progression at } 7\end{array}$ & - & - & (6) \\
\hline KISS1 & $1(\mathrm{M})$ & Gain-of-function missense & $\begin{array}{l}\text { Autosomal dominant } \\
\text { with incomplete } \\
\text { sex-dependent } \\
\text { penetrance }\end{array}$ & 1 & - & - & (7) \\
\hline MKRN3 & 89 (76F:13M) & $\begin{array}{l}\text { Loss-of-function: } \\
\text { 1. Frameshift } \\
\text { 2. Nonsense } \\
\text { 3. Missense } \\
\text { 4. Promoter deletion }\end{array}$ & $\begin{array}{l}\text { Autosomal dominant } \\
\text { with paternal } \\
\text { expression }\end{array}$ & $\begin{array}{l}\text { Female } 6.0 \\
(3.0 \text { to } 7.8) \\
\text { Male } 8.0 \\
\text { (5.9 to } 9.0)\end{array}$ & - & $\begin{array}{l}\text { Indistinguishable from } \\
\text { idiopathic CPP }\end{array}$ & $(8,15,20)$ \\
\hline DLK1 & $10(\mathrm{~F})$ & $\begin{array}{l}\text { Loss-of-function: } \\
\text { 1. Deletion } \\
\text { 2. Frameshift }\end{array}$ & $\begin{array}{l}\text { Autosomal dominant } \\
\text { with paternal } \\
\text { expression }\end{array}$ & $\begin{array}{c}5.5 \\
\text { (4.6 to } 7.0)\end{array}$ & $\begin{array}{c}7.8 \\
\text { (7.0 to } 9.0)\end{array}$ & $\begin{array}{c}\text { Overweight/obesity } \\
\text { Early type } 2 \text { diabetes } \\
\text { Hyperlipidemia } \\
\text { Polycystic ovary syndrome }\end{array}$ & $(22,23)$ \\
\hline
\end{tabular}

Both activating mutations of KISSIR and KISSI genes associated to CPP cases contributed to the elucidation of the role of kisspeptin pathway in pubertal control in physiological conditions. However, no other $\mathrm{CPP}$ cases with activating mutations in the kisspeptin pathway have been reported since then, suggesting these are very rare molecular mechanisms of CPP.

\section{Inactivating mutations in imprinted gene MKRN3 leading to familial CPP}

With the increasingly available techniques of next generation sequencing, the investigation of the genetics of CPP has further progressed. Abreu and cols. (8) first described the role of makorin RING-finger 3 ( $M K R N 3)$ in the pathogenesis of CPP in 2013. Whole exome sequencing analysis of 32 patients with CPP (27 girls and 5 boys) identified 15 individuals ( 8 girls and 7 boys) from 5 families with CPP who harbored MKRN3 inactivating mutations. Four families had frameshift mutations, while the fifth family had a missense variant that was predicted to be deleterious and causes loss-of-function (8).

MKRN3 is an intronless gene, located at the imprinted locus $15 \mathrm{qll}-\mathrm{ql} 3$, the critical region of Prader-Willi and Angelman syndromes $(9,10)$. Genomic imprinting is a physiological process of gene silencing, which is defined as the monoallelic expression of a gene according to its parental origin. Therefore imprinted genes are silenced (imprinted) in one parental allele and expressed only from the other (11). DNA methylation usually occurs in cytosine nucleotides located within
CpG islands at promoter or intergenic regions, resulting in transcriptional silencing $(9,11)$. MKRN3 presents maternal imprinting (maternal allele silencing), therefore, patients present CPP only when they inherit the mutated allele from their fathers (Figure 2) $(8,9)$. The MKRN3 gene encodes a 507 -amino acid protein with a RING $(\mathrm{C} 3 \mathrm{HC} 4)$ zing finger motif and multiple $\mathrm{C} 3 \mathrm{H}$ zinc finger motifs (9). The gene is expressed ubiquitously as a 3 -kb transcript, with a 5 -prime $\mathrm{CpG}$ island region that is methylated on the maternal allele. MKRN3 is associated with protein ubiquitination, a process in which a ubiquitin is attached to a protein, and in turn tags it for movement to the proteasome, where it is then degraded (9).

In their initial report, Abreu and cols. (8) performed real-time PCR in the arcuate nucleus of mice. In both sexes, the Mkrn3 mRNA levels were highest on postnatal days 10 and 12 and declined subsequently, reaching their nadir by days $18-22$, in the same period that the Kiss 1 and Tac2 expression were shown to increase, marking the onset of puberty (8). Therefore, it was concluded that MKRN3 had an inhibitory role on pubertal onset, and that its function loss would favor the premature stimulation of $\mathrm{GnRH}$ secretion and puberty development. This finding was corroborated by a study in Danish girls, in which circulating serum levels of MKRN3 where shown to decrease by $15 \%$ preceding pubertal onset; furthermore, MKRN3 levels were lower in early maturing girls when compared witg age-matched prepubertal girls (12). However, up to 
A

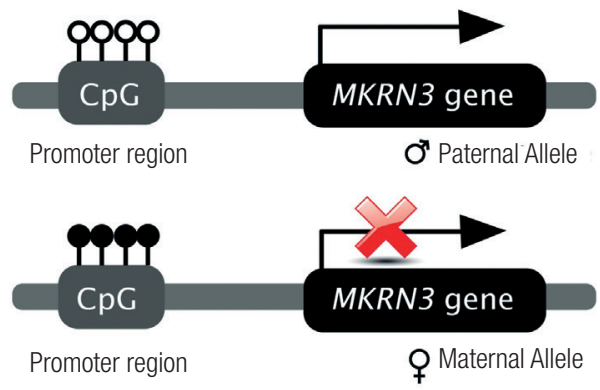

C

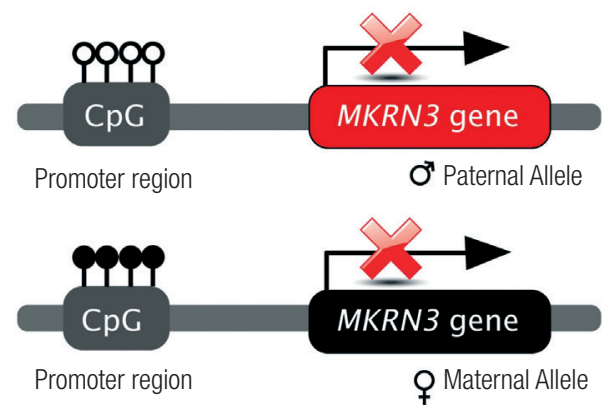

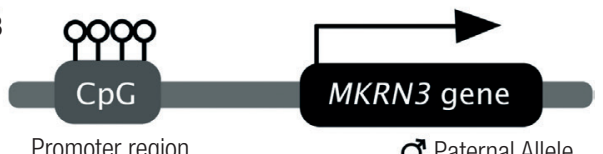

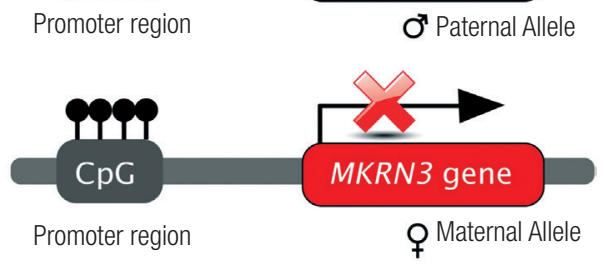

O Unmethylated

Methylated

Normal functioning gene

Loss-of function mutation

Figure 2. (A) Normal pattern of imprinting of MKRN3 gene, with silencing of the maternal allele (by methylation of its promoter region) and monoallelic expression of the paternal allele. (B) The genotype of a patient who inherited a maternal loss-of-function mutation. The patient will not present central precocious puberty, because the paternal allele is normally expressed. (C) The genotype of a patient who inherited a paternal loss-of-function mutation. The patient will present central precocious puberty because both alleles are inactive: the paternal is mutated and the maternal is silenced.

now, a cutoff value that accurately predicts puberty has not been established (13). Furthermore, the exact mechanism through which MKRN3 expression modulates GnRH secretion and puberty has not been fully elucidated, although a few factors have been recently described (14).

Subsequently, MKRN3 mutations were investigated in a large cohort comprising patients from three Brazilian university hospitals. Macedo and cols. (15) studied 215 patients with apparently sporadic CPP, identifying eight new cases of CPP caused by MKR N3 loss-of-function mutations. In another study, Bessa and cols.. (16) reported MKRN3 mutations in 8 out of 20 boys with apparently idiopathic CPP, reporting a high frequency of MKRN3 mutations in male with CPP previously classified as idiopathic. Further studies performed in centers from different countries, as well as a recent systematic review, confirmed that defects in $M K R N 3$ are the most common cause of genetic CPP, with prevalence ranging from 33 to $46 \%$ in familial cases $(15,17)$, and 0.4 to $5 \%$ in sporadic cases $(16,18-20)$.

Further evaluation through genome sequencing of 115 patients aiming to investigate possible pathogenic variants in noncoding regions revealed a rare heterozygous deletion in the proximal promoter region of MKRN3 in a girl with CPP (21). In silico analysis predicted that this variant would lead to a loss of a downstream responsive element antagonist modulator (DREAM) binding site, which would lead to a decrease in MKR N3 expression; this finding was confirmed with the in vitro reduction of the promoter activity (21).

Currently, more than 30 different loss-of-function mutations of MKRN3 have been reported since the first report by Abreu and cols. (20) (Table 1). Notably, a large proportion of these mutations were frameshift affecting the amino-terminal region of the protein that is codified by nucleotides between $476-482$, which consists of a rich poly $\mathrm{C}$ site, suggesting that this area represents a hotspot for inactivating mutations in CPP.

The clinical features of CPP caused by MKRN3 inactivating mutations are indistinguishable from other idiopathic CPP. The median age of puberty onset is 6 in girls and 8 in boys, with the youngest reported case being a 3 -year-old girl with Tanner stage 3 breast development and advanced bone age. Up to now, remarkable clinical or hormonal features distinguishing patients with CPP caused by MKRN3 mutations are not identified (8). Moreover, the response to treatment with $\mathrm{GnRH}$ agonists appears to be adequate, as outlined by Macedo and cols. (15). 


\section{Inactivating mutations in imprinted gene DLK1 leading to familial CPP}

In 2017, Dauber and cols. (22) studied a threegeneration Brazilian family with five affected members (two sisters, two paternal half-sisters, and their paternal grandmother) using linkage analysis followed by wholegenome sequencing. The children's fathers had normal pubertal timing, suggesting an autosomal dominant inheritance either with incomplete penetrance or an imprinting mechanism. A complex defect in the Deltalike 1 homolog (DLKI) imprinted gene was identified (14-kb heterozygous deletion that encompassed the first exon of the gene). Moreover, sequencing analysis showed also a 269-bp duplication from intron 3 of $D L K 1$, segregating with the deletion (22). DLKI is an imprinted paternally expressed gene, meaning that carriers of DLKI defects only manifest the phenotype if the defect is inherited from their fathers, similar to MKRN3 affected families.

Subsequently, three novel families with DLKI loss-of-function mutations with paternal expression (phenotype was expressed only when the mutation was inherited from the father) were described by Gomes and cols. (23), who performed DLKI sequencing analysis in 60 patients with CPP or history of precocious menarche. Three distinct frameshift mutations in the exon 5 of $D L K I$ were identified in five patients from three unrelated families (p.Gly199Alafs*11, p.Val271Cysfs* 14 and p.Prol60Leufs*50) (23).

DLKl, also called preadipocyte factor 1 (Pref-1), is a transmembrane protein containing epidermal growth factor-like repeats in its extracellular domain (22). It is a noncanonical ligand in the Delta-Notch signaling pathway, known to play a role in several cell types differentiation, mostly in inhibiting adipocyte differentiation $(22,24)$. DLKl has a wide expression in fetal life; however, in postnatal life, the expression decreases, except in endocrine glands (mainly in the adrenals, the pituitary and the ovaries) $(22,24)$. A hypothalamic function of DLKI has been suggested by evidence of its expression also in several hypothalamic nuclei (24).

Human DLKI is a paternally expressed gene located at chromosome $14 \mathrm{q} 32.2$, a region encompassing a cluster of imprinted genes (25). Maternal uniparental disomy, epimutations and paternal deletions at chromosome $14 \mathrm{q} 32.2$ lead to expression loss of the paternally expressed genes of this region, including
DLK1. These molecular abnormalities are associated to Temple syndrome, an imprinting disorder mainly characterized by pre- and postnatal growth failure, hypotonia, small hands and feet, CPP, and overweight/ obesity after infancy (26-28).

To investigate the effect of mutations on DLKI production, serum DLKI levels were measured in the affected family members (mutated DLKI patients) and in controls, using an available soluble DLKI enzymelinked immunosorbent assay (ELISA) $(22,23)$. Serum DLKI concentrations were undetectable in all tested affected individuals. Moreover, Abi Habib and cols. (11) identified undetectable levels of serum DLKI in Temple syndrome patients with epimutations and deletions at chromosome $14 \mathrm{q} 32.2$. Taken together, these data indicate that serum DLKl measurement may be a potential screening method for investigating DLKl deficiency in affected children.

Interestingly, patients with DLKI mutations presented higher metabolic abnormalities when compared with patients with patients with idiopathic CPP as demonstrated by Gomes and cols. (23), thus suggesting that this antiadipogenic factor represents a novel link between reproduction and metabolism. The more prevalent features at adulthood were central obesity, early onset glucose intolerance/type 2 diabetes, and hyperlipidemia. These metabolic alterations were similar to the phenotype of DlkI deficiency previously described in a null mouse model (23). In addition, patients with Temple syndrome may develop obesity, early onset type 2 diabetes and hyperlipidemia as young adults (26-28).

Another clinical aspect demonstrated by Gomes and cols. (23) was the presence of polycystic ovarian syndrome (PCOS) in two out of 10 adult women evaluated with DLKI mutations. It is postulated that a neuroendocrine component may participate in PCOS physiopathology with increased $\mathrm{GnRH}$ pulse frequency leading to increased LH production over FSH production. It is well established that girls with CPP may present an abnormality in GnRH pulses. The authors suggested that this phenomenon, as well as the poor metabolic profile, could link both conditions (23).

In addition, the DLKI protein function has been studied in distinct metabolic settings. It has been proposed that DLKI may alter the metabolic mode of the organism from lipid storage to peripheral lipid oxidation, protecting the organism from hepatic steatosis (29). Interestingly, the exogenous 
administration of DLKl in mice reduced hepatic steatosis and hyperglycemia via AMPK activation in the liver (30). Moreover, in pregnancy, it has been shown that elevated DLKl circulating levels are required to the increased maternal fatty acid metabolism. In a study evaluating late gestation, Cleaton and cols. (31) demonstrated that maternal levels of DLKI were fetusderived and could be used as a marker of complicated fetal growth restriction.

\section{Chromosomal abnormalities}

A few studies have reported rare cases of patients with idiopathic CPP associated with complex phenotypes, primarily related to clinical syndromes or chromosomal abnormalities $(1,32)$. To date, CPP has been reported as part of the phenotypic spectrum of the following genetic syndromes: 1) Temple syndrome (maternal uniparental disomy or epimutation $14 \mathrm{q} 32.2$ ) (90\% of cases) $(27,28)$; 2) Silver-Russell syndrome (maternal uniparental disomy of chromosome 7 ) (up to $25 \%$ of case) (2,33) 3) Williams-Beuren syndrome (7q11.23 deletion) (10-18\% of cases) (32); 4) Prader-Willi syndrome (15q11-q13 paternal deletion) (4-10\% of cases) (1). Additionally, studies have been reported regarding patients with idiopathic CPP associated with distinct copy number variants, such as $1 \mathrm{p} 36$ deletion, $9 \mathrm{p}$ distal deletion, $9 \mathrm{q} 34.3$ duplication (including $\mathrm{NOTCH}$ gene), Xpl1.23-pl1.22 duplication, and CDKL5 gene deletion (1). Notably, Temple syndrome, Silver-Russell syndrome, and Prader-Willi syndrome are imprinting disorders (11).

In conclusion, central precocious puberty is considered idiopathic in most children, particularly in girls. This scenario may change with the identification of genetic causes underlying CPP. Recent identifications of MKRN3 and DLKI mutations in familial CPP established the relevance of genetic factors in the physiopathology of this condition. In addition, some studies have emphasized the association of CPP with rare cases of genetic and epigenetic defects characterized by clinical syndromes or complex phenotypes. In this context, the selection of patients with CPP for genetic testing should consider the family history and segregation (family members with similar phenotype), and the presence of other clinical features (Figure 3). Genetic testing has now become a more routine clinical practice, thus allowing a greater number of patients to be properly tested.

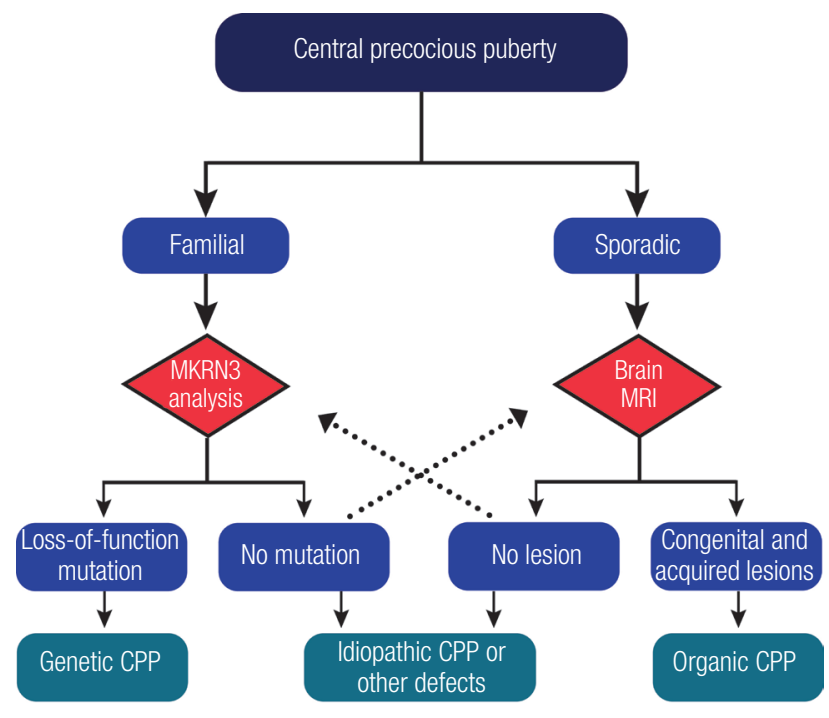

Figure 3. Flow chart for investigation of familial central precocious puberty.

It is noteworthy that patients with genetic causes of CPP have been described as presenting adequate clinical and laboratory responses to long-acting GnRH analogs (the gold standard treatment), similar to patients with idiopathic CPP. Nevertheless, careful monitoring for reproductive, metabolic, and general health related outcomes should be considered during management of patients with CPP with genetic conditions, including an extended follow-up until adulthood.

Disclosure: no potential conflict of interest relevant to this article was reported.

\section{REFERENCES}

1. Macedo DB, Silveira LF, Bessa DS, Brito VN, Latronico AC. Sexual Precocity - Genetic Bases of Central Precocious Puberty and Autonomous Gonadal Activation. Endocr Dev. 2016;29:50-71.

2. Latronico AC, Brito VN, Carel JC. Causes, diagnosis, and treatment of central precocious puberty. Lancet Diabetes Endocrinol. 2016;4(3):265-74.

3. Day FR, Thompson DJ, Helgason H, Chasman DI, Finucane $H$, Sulem $P$, et al. Genomic analyses identify hundreds of variants associated with age at menarche and support a role for puberty timing in cancer risk. Nat Genet. 2017;49(6):834-41.

4. de Vries L, Kauschansky A, Shohat M, Phillip M. Familial central precocious puberty suggests autosomal dominant inheritance. J Clin Endocrinol Metab. 2004;89(4):1794-800.

5. Teilmann G, Pedersen CB, Jensen TK, Skakkebaek NE, Juul A. Prevalence and incidence of precocious pubertal development in Denmark: an epidemiologic study based on national registries. Pediatrics. 2005;116(6):1323-8.

6. Teles MG, Bianco SD, Brito VN, Trarbach EB, Kuohung W, Xu $\mathrm{S}$, et al. A GPR54-activating mutation in a patient with central precocious puberty. N Engl J Med. 2008;358(7):709-15.

7. Silveira LG, Noel SD, Silveira-Neto AP, Abreu AP, Brito VN, Santos MG, et al. Mutations of the KISS1 gene in disorders of puberty. J Clin Endocrinol Metab. 2010;95(5):2276-80. 
8. Abreu AP, Dauber A, Macedo DB, Noel SD, Brito VN, Gill JC, et al. Central precocious puberty caused by mutations in the imprinted gene MKRN3. N Engl J Med. 2013;368(26):2467-75.

9. Jong MT, Gray TA, Ji Y, Glenn CC, Saitoh S, Driscoll DJ, et al. A novel imprinted gene, encoding a RING zinc-finger protein, and overlapping antisense transcript in the Prader-Willi syndrome critical region. Hum Mol Genet. 1999;8(5):783-93.

10. Nicholls RD, Saitoh S, Horsthemke B. Imprinting in Prader-Willi and Angelman syndromes. Trends Genet. 1998;14(5):194-200.

11. Abi Habib W, Brioude F, Azzi S, Rossignol S, Linglart A, Sobrier $M L$, et al. Transcriptional profiling at the DLK1/MEG3 domain explains clinical overlap between imprinting disorders. Sci Adv. 2019;5(2):eaau9425.

12. Hagen $C P$, Sørensen $K$, Mieritz MG, Johannsen TH, Almstrup $K$, Juul A. Circulating MKRN3 levels decline prior to pubertal onset and through puberty: a longitudinal study of healthy girls. J Clin Endocrinol Metab. 2015;100(5):1920-6.

13. Busch AS, Hagen $C P$, Almstrup $K$, Juul A. Circulating MKRN3 Levels Decline During Puberty in Healthy Boys. J Clin Endocrinol Metab. 2016;101(6):2588-93.

14. Yellapragada V, Liu X, Lund C, Känsäkoski J, Pulli K, Vuoristo S, et al. MKRN3 Interacts With Several Proteins Implicated in Puberty Timing but Does Not Influence. Front Endocrinol (Lausanne). 2019;10:48.

15. Macedo DB, Abreu AP, Reis AC, Montenegro LR, Dauber A, Beneduzzi $D$, et al. Central precocious puberty that appears to be sporadic caused by paternally inherited mutations in the imprinted gene makorin ring finger 3. J Clin Endocrinol Metab. 2014;99(6):E1097-103.

16. Bessa DS, Macedo DB, Brito VN, França MM, Montenegro LR, Cunha-Silva $M$, et al. High Frequency of MKRN3 Mutations in Male Central Precocious Puberty Previously Classified as Idiopathic. Neuroendocrinology. 2017;105(1):17-25.

17. Simon D, Ba I, Mekhail N, Ecosse E, Paulsen A, Zenaty D, et al. Mutations in the maternally imprinted gene MKRN3 are common in familial central precocious puberty. Eur J Endocrinol. 2016;174(1):1-8.

18. Lee HS, Jin HS, Shim YS, Jeong HR, Kwon E, Choi V, et al. Low Frequency of MKRN3 Mutations in Central Precocious Puberty Among Korean Girls. Horm Metab Res. 2016;48(2):118-22.

19. Ortiz-Cabrera NV, Riveiro-Álvarez R, López-Martínez M, PérezSegura P, Aragón-Gómez I, Trujillo-Tiebas MJ, et al. Clinical Exome Sequencing Reveals MKRN3 Pathogenic Variants in Familial and Nonfamilial Idiopathic Central Precocious Puberty. Horm Res Paediatr. 2017;87(2):88-94.

20. Valadares LP, Meireles CG, De Toledo IP, Santarem de Oliveira R, Gonçalves de Castro LC, Abreu AP, et al. MKRN3 Mutations in Central Precocious Puberty: A Systematic Review and MetaAnalysis. J Endocr Soc. 2019;3(5):979-95.
21. Macedo DB, França MM, Montenegro LR, Cunha-Silva M, Best DS, Abreu AP, et al. Central Precocious Puberty Caused by a Heterozygous Deletion in the MKRN3 Promoter Region. Neuroendocrinology. 2018;107(2):127-32.

22. Dauber A, Cunha-Silva M, Macedo DB, Brito VN, Abreu AP, Roberts SA, et al. Paternally Inherited DLK1 Deletion Associated With Familial Central Precocious Puberty. J Clin Endocrinol Metab. 2017;102(5):1557-67.

23. Gomes LG, Cunha-Silva M, Crespo RP, Ramos CO, Montenegro LR, Canton A, et al. DLK1 is a novel link between reproduction and metabolism. J Clin Endocrinol Metab. 2019;104(6):2112-2120.

24. Villanueva $\mathrm{C}$, Jacquier $\mathrm{S}$, de Roux N. DLK1 is a somato-dendritic protein expressed in hypothalamic arginine-vasopressin and oxytocin neurons. PLoS One. 2012;7(4):e36134.

25. Schmidt JV, Matteson PG, Jones BK, Guan XJ, Tilghman SM. The Dlk1 and Gtl2 genes are linked and reciprocally imprinted. Genes Dev. 2000;14(16):1997-2002.

26. Ioannides Y, Lokulo-Sodipe K, Mackay DJ, Davies JH, Temple IK. Temple syndrome: improving the recognition of an underdiagnosed chromosome 14 imprinting disorder: an analysis of 51 published cases. J Med Genet. 2014;51(8):495-501.

27. Kagami M, Nagasaki K, Kosaki R, Horikawa R, Naiki Y, Saitoh S, et al. Temple syndrome: comprehensive molecular and clinical findings in 32 Japanese patients. Genet Med. 2017;19(12):135666.

28. Geoffron S, Abi Habib W, Chantot-Bastaraud S, Dubern B, Steunou V, Azzi S, et al. Chromosome 14q32.2 Imprinted Region Disruption as an Alternative Molecular Diagnosis of Silver-Russell Syndrome. J Clin Endocrinol Metab. 2018;103(7):2436-46.

29. Charalambous M, Da Rocha ST, Radford EJ, Medina-Gomez G, Curran S, Pinnock SB, et al. DLK1/PREF1 regulates nutrient metabolism and protects from steatosis. Proc Natl Acad Sci U S A. 2014;111(45):16088-93.

30. Lee $\mathrm{YH}$, Yun MR, Kim HM, Jeon BH, Park BC, Lee BW, et al. Exogenous administration of DLK1 ameliorates hepatic steatosis and regulates gluconeogenesis via activation of AMPK. Int $\mathrm{J}$ Obes (Lond). 2016;40(2):356-65.

31. Cleaton MA, Dent CL, Howard M, Corish JA, Gutteridge I, Sovio $\mathrm{U}$, et al. Fetus-derived DLK1 is required for maternal metabolic adaptations to pregnancy and is associated with fetal growth restriction. Nat Genet. 2016;48(12):1473-80.

32. Canton A, Brito V, Montenegro L, Ramos C, Macedo D, Bessa D, et al. Clinical and Genetic Features of Central Precocious Puberty Associated with Complex Phenotypes. Horm Res Paediatr. 2018:98-9.

33. Canton APM, Harbison M, Abi Habib W, Salem J, Blaise A, Geoffron S, et al. Clinical and molecular analysis of pubertal control in a cohort of Silver-Russell and Temple syndrome patients. Horm Res Paediatr. 2017:9. 